\title{
Reassortant Clade 2.3.4.4 of Highly Pathogenic Avian Influenza A(H5N6) Virus, Taiwan, 2017
}

\section{Li-Hsuan Chen, ${ }^{1}$ Dong-Hun Lee, ${ }^{1}$ Yu-Pin Liu, Wan-Chen Li, David E. Swayne, Jen-Chieh Chang, Yen-Ping Chen, Fan Lee, Wen-Jane Tu, Yu-Ju Lin}

Author affiliations: Council of Agriculture, New Taipei City, Taiwan (L.-H. Chen, Y.-P. Liu, W.-C. Li, J.-C. Chang, Y.-P. Chen, F. Lee, W.-J. Tu, Y.-J. Lin); US Department of Agriculture, Athens, Georgia, USA (D.-H. Lee, D.E. Swayne)

DOI: https://doi.org/10.3201/eid2406.172071

A highly pathogenic avian influenza $\mathrm{A}(\mathrm{H} 5 \mathrm{~N} 6)$ virus of clade 2.3.4.4 was detected in a domestic duck found dead in Taiwan during February 2017. The endemic situation and continued evolution of various reassortant highly pathogenic avian influenza viruses in Taiwan warrant concern about further reassortment and a fifth wave of intercontinental spread.

Since 1996, H5 A/goose/Guangdong/1/1996 (Gs/GD) $\checkmark$ lineage of highly pathogenic avian influenza viruses (HPAIVs) originating in Asia have caused outbreaks in Asia, Europe, Africa, and North America (1). The H5N1 Gs/GD lineage of HPAIV has evolved into 10 genetically distinct virus clades (0-9) and multiple subclades, including novel H5 clade 2.3.4.4 viruses, which emerged in China (2) and have evolved into 4 distinct genetic groups (2.3.4.4A-D) (3). Four 4 intercontinental waves of Gs/ GD lineage HPAIV transmission have occurred: clade 2.2 H5N1 in 2005, clade 2.3.2.1c H5N1 in 2009, clade 2.3.4.4A H5Nx in 2014, and clade 2.3.4.4B H5Nx in 2016 (4). The clade 2.3.4.4A and B H5N8 viruses spread intercontinentally; clade 2.3.4.4A caused outbreaks in Asia, Europe, and North America during 2014-2015, and clade 2.3.4.4B H5N8 caused outbreaks in Asia, Europe, and Africa during 2016-2017 (1,5). In fall 2016, clade 2.3.4.4C H5N6 viruses caused outbreaks in South Korea and Japan (6). Six distinct genotypes of clade 2.3.4.4C H5N6 viruses (designated as C1-C6) were identified in South Korea and Japan during these outbreaks; these genotypes contain different polymerase acidic and nonstructural genes from low pathogenicity influenza viruses from Eurasia $(7,8)$.

We report HPAIV H5N6 detection from a meattype duck in Taiwan in February 2017. One dead young Pekin-type domestic duck was found on a country road near the Xiuguluan River in Hualien County during wild bird and habitat surveillance for HPAIV by the Wild Bird
Society of Taipei; the carcass was forwarded to the national laboratory of the Animal Health Research Institute (online Technical Appendix 1 Figure 1, https://wwwnc. cdc.gov/EID/article/24/6/17-2071-Techapp1.pdf). We conducted complete genome sequencing and comparative phylogenetic analysis of the detected virus, A/duck/ Taiwan/1702004/2017(H5N6) (Dk/Tw/17), to trace the origin and understand its genetic features.

We detected $\mathrm{Dk} / \mathrm{Tw} / 17$ virus by using reverse transcription PCR and isolated the virus by using egg inoculation as described previously (9). We conducted an intravenous pathogenicity index test according to the World Organisation for Animal Health Manual of Diagnostic Tests and Vaccines for Terrestrial Animals (http://www.oie. int/en/international-standard-setting/terrestrial-manual). We performed full-length genome sequencing by using reverse transcription PCR amplification and Sanger sequencing (9). We estimated maximum-likelihood phylogenies by using RAxML (10) and constructed a median-joining phylogenetic network of the hemagglutinin gene by using NETWORK 5.0 (online Technical Appendix).

We classified Dk/Tw/17 as an HPAIV on the basis of the amino acid sequence at the hemagglutinin cleavage site (PLRERRRKR/G) and its high lethality in chickens (intravenous pathogenicity index 3.0). Necropsy and histologic examination revealed virus-specific necrotic and inflammatory lesions in the pancreas, heart, and brain (online Technical Appendix 1 Figure 2). Phylogenetic analyses suggested that the $\mathrm{Dk} / \mathrm{Tw} / 17$ virus belongs to clade $2.3 .4 .4 \mathrm{C}$ genotype C5 that was found in China, South Korea, and Japan during 2016-2017 (Figure, panel A; online Technical Appendix 1 Figure 1). This virus genotype acquired its polymerase acidic gene of low pathogenicity influenza viruses from Eurasia; its other genes originated in the G1.1.9 and G1.1-like lineages of H5N6 viruses from China $(7,8)$. All 8 gene segments shared high levels of nucleotide identity (99.3\%-99.9\%) with H5N6 viruses identified from wild birds in Japan and South Korea in November 2016, including A/whooper swan/Korea/Gangjin 49-1/2016 (H5N6), A/spot billed duck/ Korea/WB141/2016 (H5N6), and A/teal/Tottori/2/2016 (H5N6) (online Technical Appendix 1 Table). These viruses consistently clustered together with high bootstrap value $(>70)$ in maximum-likelihood phylogenies across all 8 gene segments (online Technical Appendix Figures 3-10).

The genotype C5 comprises 17 H5N6 HPAIVs identified from wild waterfowl in China, Japan, and South Korea during November-December 2016; a virus identified from a chicken farm (A/chicken/Korea/H23/2016 [H5N6]) in South Korea in November 2016; and the Dk/Tw/17 virus. Genotype C5 is phylogenetically distinct from viruses that caused outbreaks in poultry farms in Japan and South

${ }^{1}$ These authors contributed equally to this article. 


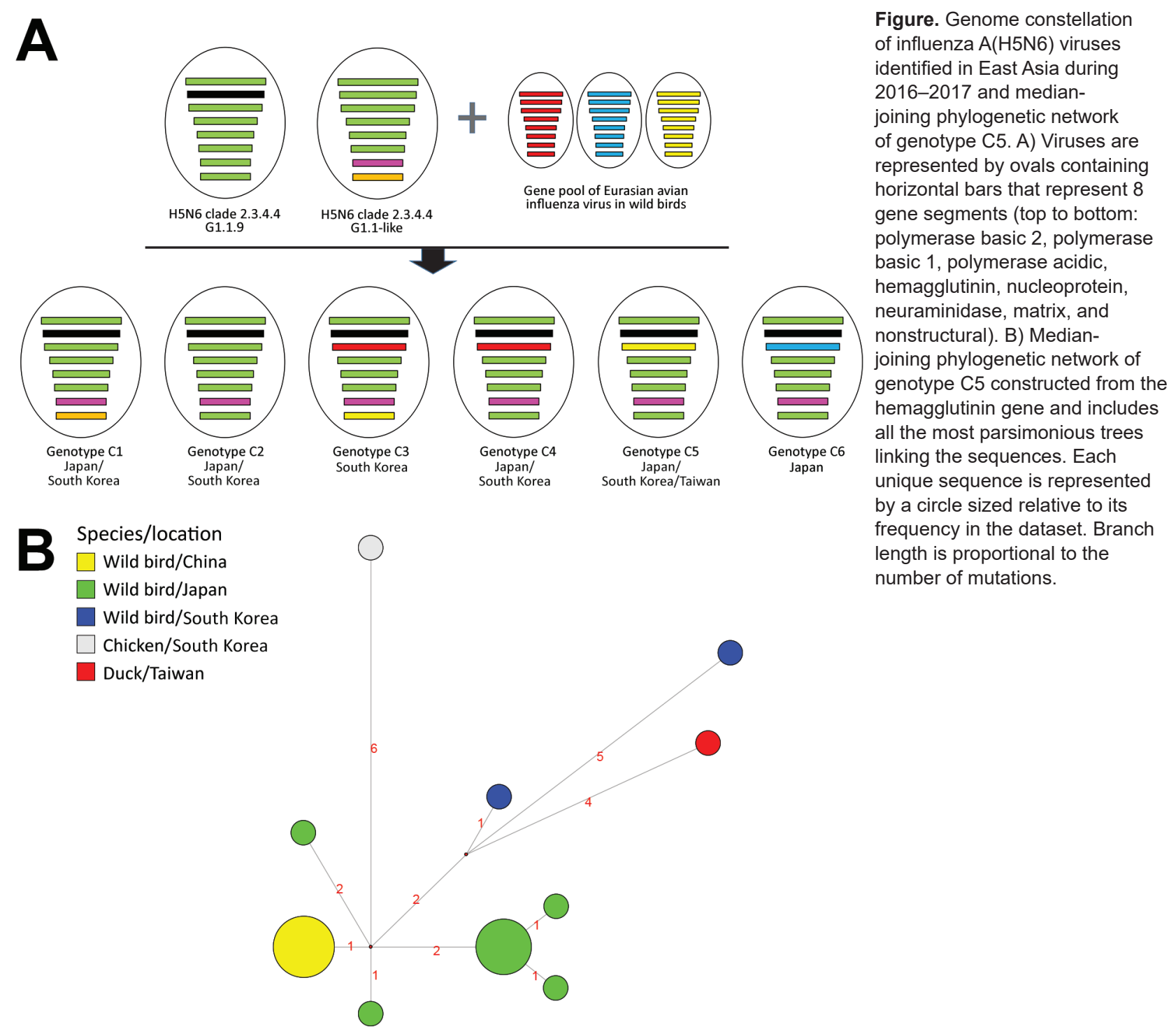

Korea during 2016-2017. This genotype has independently evolved and been maintained in wild bird populations in the bird flyway of East Asia, highlighting how wild waterfowl play an important role in the maintenance and dissemination of this HPAIV. In addition, the median-joining phylogenetic network analysis suggests that the A/chicken/ Korea/H23/2016 (H5N6) is not the direct ancestor of the $\mathrm{Dk} / \mathrm{Tw} / 17$ virus, which was likely caused by separate introduction from wild birds (Figure, panel B).

The site where the dead duck was collected is adjacent to a river and located near many ponds used for duck farming. After identification of $\mathrm{Dk} / \mathrm{Tw} / 17$, intensified active surveillance conducted over 3 months detected additional clade 2.3.4.4C H5N6 HPAIVs from 12 farms in 4 counties (online Technical Appendix 1 Figure 1). Clade 2.3.4.4A H5Nx HPAIVs, mainly H5N2 and H5N8, have caused outbreaks in the poultry industry of Taiwan since January 2015
(9). In 2017, clade 2.3.4.4A H5Nx and 2.3.4.4C H5N6 HPAIVs were detected in domestic poultry. The endemic situation and continued evolution of various reassortant HPAIVs in domestic poultry warrants concern about further reassortment. Enhanced active surveillance in domestic and wild waterfowl is required to monitor the spread and onward reassortment in Taiwan and to inform the design of improved prevention and control strategies.

\section{Acknowledgments}

We thank the Wild Bird Society of Taipei for submitting cases and contributing to the early detection of disease. Acknowledgments for laboratory contributions by GISAID partners are listed in online Technical Appendix 2 (https://wwwnc.cdc.gov/EID/article/24/6/17-2071-Techapp2.xlsx).

This work was funded by the Council of Agriculture of Taiwan. 


\begin{abstract}
About the Author
Dr. Li-Hsuan Chen is an assistant research fellow at the Epidemiology Division, Animal Health Research Institute, New Taipei City, Taiwan. Her main research interests include diagnosis of poultry diseases and the phylogenetic and phenotypic characterization of avian influenza viruses.
\end{abstract}

\section{References}

1. Lee DH, Bertran K, Kwon JH, Swayne DE. Evolution, global spread, and pathogenicity of highly pathogenic avian influenza H5Nx clade 2.3.4.4. J Vet Sci. 2017;18(S1):269-80. http://dx.doi.org/10.4142/jvs.2017.18.S1.269

2. Zhao G, Gu X, Lu X, Pan J, Duan Z, Zhao K, et al. Novel reassortant highly pathogenic $\mathrm{H} 5 \mathrm{~N} 2$ avian influenza viruses in poultry in China. PLoS One. 2012;7:e46183. http://dx.doi.org/ 10.1371/journal.pone.0046183

3. Lee DH, Bahl J, Torchetti MK, Killian ML, Ip HS, DeLiberto TJ, et al. Highly pathogenic avian influenza viruses and generation of novel reassortants, United States, 2014-2015. Emerg Infect Dis. 2016;22:1283-5. http://dx.doi.org/10.3201/ eid2207.160048

4. Sims L, Harder T, Brown I, Gaidet N, Belot G. Dobschuetz Sv, et al. Highly pathogenic H5 avian influenza in 2016 and 2017observations and future perspectives [cited 2017 Dec 15]. http://agritrop.cirad.fr/585953

5. Global Consortium for H5N8 and Related Influenza Viruses. Role for migratory wild birds in the global spread of avian influenza H5N8. Science. 2016;354:213-7. http://dx.doi.org/ $10.1126 /$ science.aaf 8852

6. Okamatsu M, Ozawa M, Soda K, Takakuwa H, Haga A, Hiono T, et al. Characterization of highly pathogenic avian influenza virus A(H5N6), Japan, November 2016. Emerg Infect Dis. 2017;23: 691-5. http://dx.doi.org/10.3201/eid2304.161957

7. Takemae N, Tsunekuni R, Sharshov K, Tanikawa T, Uchida Y, Ito $\mathrm{H}$, et al. Five distinct reassortants of H5N6 highly pathogenic avian influenza A viruses affected Japan during the winter of 2016-2017. Virology. 2017;512:8-20. http://dx.doi.org/10.1016/ j.virol.2017.08.035

8. Lee EK, Song BM, Lee YN, Heo GB, Bae YC, Joh SJ, et al. Multiple novel H5N6 highly pathogenic avian influenza viruses, South Korea, 2016. Infect Genet Evol. 2017;51:21-3. http://dx.doi.org/10.1016/j.meegid.2017.03.005

9. Lee MS, Chen LH, Chen YP, Liu YP, Li WC, Lin YL, et al. Highly pathogenic avian influenza viruses $\mathrm{H} 5 \mathrm{~N} 2, \mathrm{H} 5 \mathrm{~N} 3$, and H5N8 in Taiwan in 2015. Vet Microbiol. 2016;187:50-7. http://dx.doi.org/10.1016/j.vetmic.2016.03.012

10. Stamatakis A. RAxML version 8: a tool for phylogenetic analysis and post-analysis of large phylogenies. Bioinformatics. 2014;30:1312-3. http://dx.doi.org/10.1093/ bioinformatics/btu033

Address for correspondence: Yu-Ju Lin, Animal Health Research Institute, Division of Epidemiology, No. 376 Zhongzheng Rd, Danshui District, New Taipei City 251, Taiwan; email: yjlin@mail.nvri.gov.tw

\section{Reemergence of Human Monkeypox in Nigeria, 2017}

\author{
Adesola Yinka-Ogunleye, Olusola Aruna, \\ Dimie Ogoina, Neni Aworabhi, Womi Eteng, \\ Sikiru Badaru, Amina Mohammed, \\ Jeremiah Agenyi, E.N. Etebu, \\ Tamuno-Wari Numbere, Adolphe Ndoreraho, \\ Eduard Nkunzimana, Yahyah Disu, \\ Mahmood Dalhat, Patrick Nguku, \\ Abdulaziz Mohammed, Muhammad Saleh, \\ Andrea McCollum, Kimberly Wilkins, \\ Ousmane Faye, Amadou Sall, Christian Happi, \\ Nwando Mba, Olubumi Ojo, Chikwe Ihekweazu
}

Author affiliations: Nigeria Centre for Disease Control, Abuja, Nigeria (A. Yinka-Ogunleye, O. Aruna, W. Eteng, S. Badaru, Amina Mohammed, J. Agenyi, N. Mba, O. Ojo, C. Ihekweazu); Measure Evaluation, Abuja (O. Aruna); Niger Delta University Teaching Hospital/Niger Delta University, Yenagoa, Nigeria (D. Ogoina); Bayelsa State Ministry of Health, Yenagoa (N. Aworabhi, E.N. Etebu); Nigeria Field Epidemiology and Laboratory Training Programme, Abuja (T.-W. Numbere, A. Ndoreraho, E. Nkunzimana, Y. Disu); African Field Epidemiology Network, Abuja (M. Dalhat, P. Nguku); Africa Centres for Disease Control and Prevention, Addis Ababa, Ethiopia (Abdulaziz Mohammed); US Centers for Disease Control and Prevention, Atlanta, Georgia, USA (M. Saleh, A. McCollum, K. Wilkins); Institut Pasteur, Dakar, Senegal (O. Faye, A. Sall); Redeemers University, Ede, Nigeria (C. Happi)

DOI: https://doi.org/10.3201/eid2406.180017

In Nigeria, before 2017 the most recent case of human monkeypox had been reported in 1978. By mid-November 2017 , a large outbreak caused by the West African clade resulted in 146 suspected cases and 42 laboratory-confirmed cases from 14 states. Although the source is unknown, multiple sources are suspected.

$\mathrm{H}$ uman monkeypox is a rare zoonotic infection caused by an orthopoxvirus and characterized by smallpoxlike signs and symptoms (1). The disease is endemic to the Democratic Republic of the Congo. Reported outbreaks have occurred mainly in rural rainforest areas of the Congo basin and West Africa, caused by the Central and West African clades of the virus, respectively $(1-6)$. The West African clade is associated with milder disease, fewer deaths, and limited human-to-human transmission. Since 1970, only $\approx 10$ cases in West Africa had been reported; in 2003, a total of 81 cases ( $41 \%$ laboratory confirmed) were reported in the United States $(2,7,8)$. In Nigeria, a case of human monkeypox in a 4-year-old child in the southeastern part 